\title{
AXAF VETA-I Mirror Encircled Energy Measurements and Data Reduction
}

\author{
Ping Zhao, Mark D. Freeman, John P. Hughes, Edwin M. Kellogg, and Dan T. Nguyen \\ Harvard-Smithsonian Center for Astrophysics \\ 60 Garden Street, Cambridge, MA 02138 \\ Marshall Joy and Jeffery J. Kolodziejczak \\ ES-65, Marshall Space Flight Center, AL 35812
}

\begin{abstract}
The AXAF VETA-I mirror encircled energy was measured with a series of apertures and two flow gas proportional counters at five X-ray energies ranging from 0.28 to $2.3 \mathrm{keV}$. The proportional counter has a thin plastic window with an opaque wire mesh supporting grid. Depending on the counter position, this mesh can cause the X-ray transmission to vary as much as $\pm 9 \%$, which directly translates into an error in the encircled energy. In order to correct this wire mesh effect, window scan measurements were made, in which the counter was scanned in both horizontal $(Y)$ and vertical $(Z)$ directions with the aperture fixed. Post VETA measurement of the VXDS setup were made to determine the exact geometry and position of the mesh grid. Computer models of the window mesh were developed to simulate the X-ray transmission based on this measurement. The window scan data were fitted to such mesh models and corrections were made. After this study, the mesh effect was well understood and the final results of the encircled energy were obtained with an uncertainty of less than $0.8 \%$.
\end{abstract}

\section{INTRODUCTION}

The Verification Engineering Test Article-I (VETA-I), made of Zerodur with a diameter of 1.2 meters, is the uncoated outmost mirror pair of The Advanced X-ray Astrophysical Facility (AXAF), the third of NASA's four Great Space Observatories. ${ }^{1}$ Its mirror figures and surface quality were measured at the X-ray Calibration Facility (XRCF) of the Marshall Space Flight Center (MSFC) from August to October of $1991{ }^{2}$ $\mathrm{X}$-rays generated by an electron impact source ${ }^{3} 528$ meters away were focused by the VETA to its focal plane, which is 10 meters behind the VETA. The focused X-rays were then detected and measured with the VETA X-ray Detecting System (VXDS) built by SAO. ${ }^{4}$ Four types of results were obtained, i.e. Full Width Half Maximum (FWHM), Encircled Energy, Effective Area, and Ring Focus. All the measurements were done with X-ray detectors placed in the focal plane, except the Ring Focus measurements, which were done in the Ring focal plane (about $21 \mathrm{~mm}$ in front of the focal plane).

We first give some definitions:

Total Effective Area (or simply Effective Area): The total power reflected by the VETA into $2 \pi$ steradians, measured in units of $\mathrm{cm}^{2}$. It is equal to the projected area of an equivalent mirror with $100 \%$ reflectivity, or a projected area of $P 1 \times R^{2}(E, \theta)$, where $P 1$ is the Paraboloid mirror of the VETA and $R$ is the mirror reflectivity as a function of $\mathrm{X}$-ray energy and grazing angle.

Encircled Energy: The fraction of the power reflected from the mirror that passes through an aperture of radius $r$, in the focal plane. It is measured in units of the effective area or $\mathrm{cm}^{2}$ as a function of $r$. Therefore it is also called encircled effective area.

Fractional Encircled Energy: The Encircled Energy normalized to that contained in the largest 
aperture used for the measurement, $20 \mathrm{~mm}$ diameter $(=6.88 \mathrm{arcmin})$.

The requirements for the VETA-I test was to measure the FWHM (expected to be better than 0.5 arcsec) to \pm 0.05 arcsec precision, the Fractional Encircled Energy to $\pm 2 \%$, and the Effective Area to $\pm 5 \%$.

In this paper we discuss the VETA encircled energy and effective area measurements and their data reduction for up to the $20 \mathrm{~mm}$ diameter aperture. The total effective area involves wing scan measurement and is discussed in another paper in this volume. ${ }^{5}$ Two major corrections were made to the raw data. The first is the spectrum correction with which we take care of the spectrum contamination taken by the proportional counter. The second, an even bigger correction, is the counter window mesh correction. The first correction is discussed by another paper in this volume. ${ }^{6}$ This paper mainly deals with the second correction.

In the following sections we discuss the techniques used for the measurements, the method used for the wire mesh correction, and the results. Section 2 describes the VETA encircled energy measurements and explains the X-ray detector window mesh effect. Section 3 is about the Post VETA Measurement. Section 4 discusses the computer mesh models. Section 5 gives the mesh effect correction. And section 6 lists the final results of the VETA encircled energy.

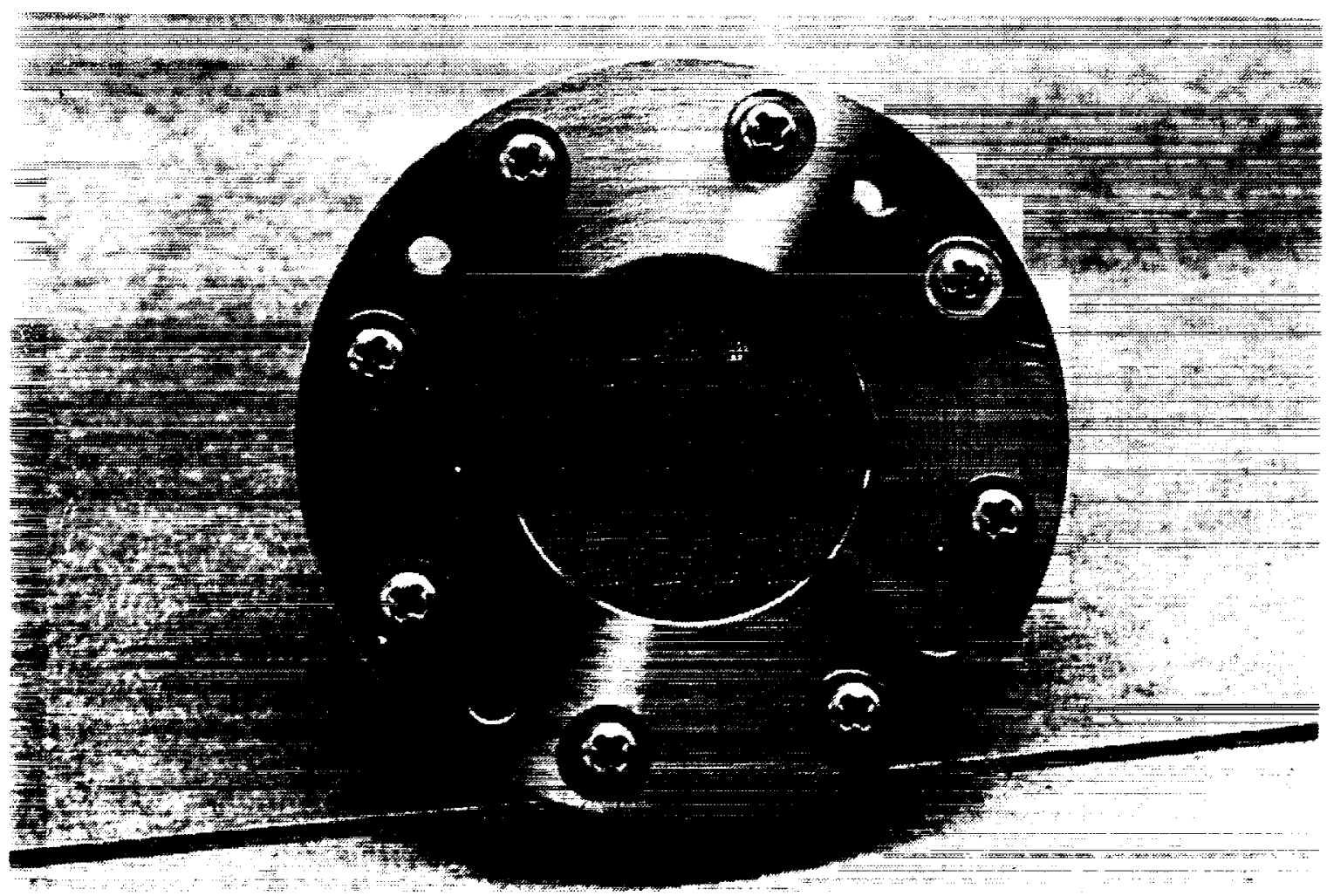

Figure 1: VXDS Flow Proportional Counter Window. The mesh supporting grid is made of gold wire with $50.8 \mu \mathrm{m}$ diameter and $529.17 \mu \mathrm{m}$ pitch.

\section{VETA-I ENCIRCLED ENERGY MEASUREMENTS AND WIRE MESH EFFECT}

VETA encircled energy measurements were made at five different $\mathrm{X}$-ray energies, i.e. $\mathrm{C}-\mathrm{K}(0.277 \mathrm{keV})$, $\mathrm{Cu}-\mathrm{L}(0.932 \mathrm{keV}), \mathrm{Al}-\mathrm{K}(1.488 \mathrm{keV}), \mathrm{Zr}-\mathrm{L}(2.067 \mathrm{keV})$ and Mo-L $(2.334 \mathrm{keV})$. The X-rays focused by the VETA pass through an aperture of radius $r$, in the focal plane, and detected by a flow gas proportional 
counter placed $25 \mathrm{~mm}$ behind the focal plane. There are 16 apertures with diameters of $0.005,0.01,0.025$, $0.05 .0 .1,0.3,0.5,0.75,1,1.5,2,3,5,7.5,10,20 \mathrm{~mm}$. The aperture 0.005 through $0.5 \mathrm{~mm}$ are laser drilled pinholes on $99.9 \%$ pure and 12.5 microns thick gold foil. The $0.75 \mathrm{~mm}$ or larger apertures were machine drilled on aluminum plate with irridite coating. We have taken the scanning electron microscope pictures of the laser drilled pinholes. Their actual sizes and shapes are slightly different from a perfect circle with quoted diameters, which is discussed by another paper in this volume. ${ }^{4}$ Each aperture was placed in the focal plane of the VETA-I and centered on the peak of the VETA Point Spread Function (PSF). The photon counts, counted by the flow gas proportional counter (also called X-ray Detection Assembly counter or XDA counter), through each aperture then represents the integral of the PSF out to the radius of that aperture. The encircled energy is measured by comparing the counting rate to that obtained in an identical flow counter, the Beam Normalization Detector (BND), of very well known area, exposed to the same incident beam in the entrance plane of the P1. Depending on the source intensity, the integration time was chosen to ensure both detectors receiving enough counts so the statistical error is less than $1 \%$. The VETA effective area is calculated as

$$
\text { Effective Area }(r)=\frac{\text { XDA Counts }(r)}{\text { BND counts }} \times \text { BND area }
$$

where BND area $=\pi \mathrm{cm}^{2}$ with an uncertainty of $0.05 \%$.

The flow proportional counter is filled with either 125 torr methane for a low energy line (C-K) or 400 torr $\mathrm{P} 10$ gas (10\% methane and $90 \%$ argon) for higher energy lines ( $\mathrm{Cu}-\mathrm{L}, \mathrm{AI}-\mathrm{K}, \mathrm{Zr}-\mathrm{L}$ and $\mathrm{Mo}-\mathrm{L}$ ). It has a thin polypropylene window with an opaque wire mesh supporting grid which prevents the window from breaking under differential pressure. The wire is made of gold with $50.8 \mu \mathrm{m}$ diameter and the average mesh period is $529.17 \mu \mathrm{m}$ (see Figure 1). When the window is uniformly illuminated, which is the case for the BND counter, the transmission is $81.72 \%$ due to the wire mesh effect. But it is not so simple for the XDA counter. As shown in Figure 2, the X-ray photons from the focal point expand into a ring of about $3 \mathrm{~mm}$ diameter when they strike the counter window, which is located $25 \mathrm{~mm}$ behind the focal plane. Depending

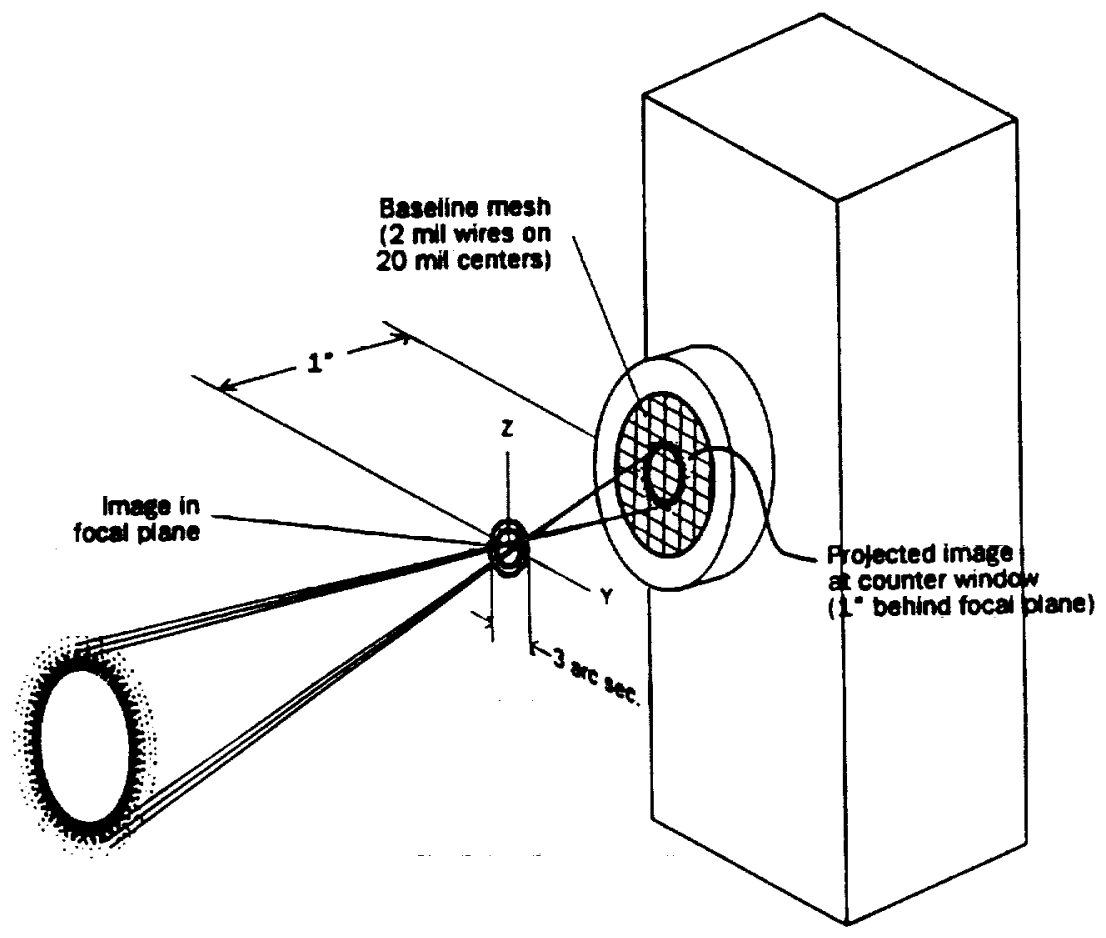

Figure 2: Counter Window Wire Mesh Effect. The X-ray photons form a ring pattern when they enter the counter window. Depending on the phase of the mesh grid relative to the photon ring, the X-ray transmission can vary from $75 \%$ to $92 \%$. 
WINDOW SCAN MEASUREMENTS

$0.600 \mathrm{~mm}$ scan $=9$ steps $\times 0.075 \mathrm{~mm}$ stepsize

or $=11$ steps $\times 0.060 \mathrm{~mm}$ siepsize

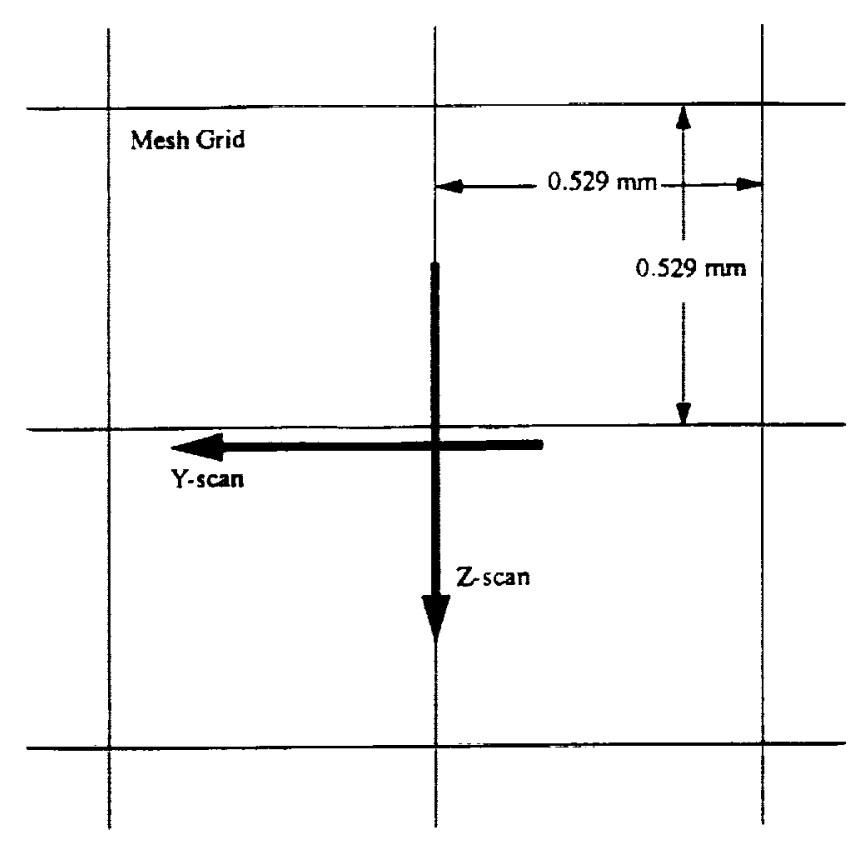

Figure 3: The planned VETA Encircled Energy Window Scan Measurement. The arrows indicate the $Y$ and $\mathrm{Z}$ positions of the aperture center with respect to that of the mesh grid. The transmission maximum occurs when the aperture center is at one of the mesh grid intersection, except for the $20 \mathrm{~mm}$ aperture, in which the transmission minimum occurs. The scan maximum occurs when the aperture center crosses one of the mesh wire, also except for the $20 \mathrm{~mm}$ aperture, in which the transmission minimum occurs.

on the phase of the mesh grid relative to the photon ring, the X-ray transmission can vary from $75 \%$ to $92 \%$, which directly translates into an error of the encircled energy. Obviously we had to take this mesh effect very seriously in order to fulfill the measurement precision requirements.

To measure the counting rate modulation due to the wire mesh and to make appropriate transmission corrections, the window was scanned in both $\mathrm{Y}$ and $\mathrm{Z}$ directions with the aperture fixed. Three sources ( $\mathrm{Al}, \mathrm{C}$ and $\mathrm{Zr}$ ) and 11 apertures $(0.3$ through $20 \mathrm{~mm}$ ) were used for the window scan measurements. The procedure was to: 1) make a scan in $Y$ direction ; 2) find the location with the highest counts; 3 ) offset the counter to this location; 4) make a scan in $Z$ direction. Each scan is a $600 \mu \mathrm{m}$ span with 9 or 11 steps (see Figure 3). Integration time is chosen to ensure enough counts $(>10000)$ for each step. Figure 4 is the Quick-look data of some typical scans, generated during the VETA test. The mesh modulation effect is clearly seen. Figure 5 is the Quick-look data for 2 and $10 \mathrm{~mm}$ apertures which shows something we could not understand at that time: the window Z-scans have much higher counts than the Y-scans. Obviously they were not done as planned because otherwise the highest point of the Y-scan curve should agree with the middle point of the Z-scan curve. Figure 6 shows all the quick-look window scan data for the Al-K line. It shows the $20 \mathrm{~mm}$ aperture window scan and the Z-scans for the 2 and $10 \mathrm{~mm}$ aperture are distinctively higher than the rest of the window scans. Compared with the wire mesh models (see Section 5), a simple analysis (we leave this to our reader as a little fun exercise) concludes that all the window scans were not done as planned. This was caused by a computer error and it complicated the data reduction. In order to make the mesh correction, we had to fully understand how the window scan measurements were actually done and the exact geometry of the apertures and counter setup. Therefore we planned the Post VETA 


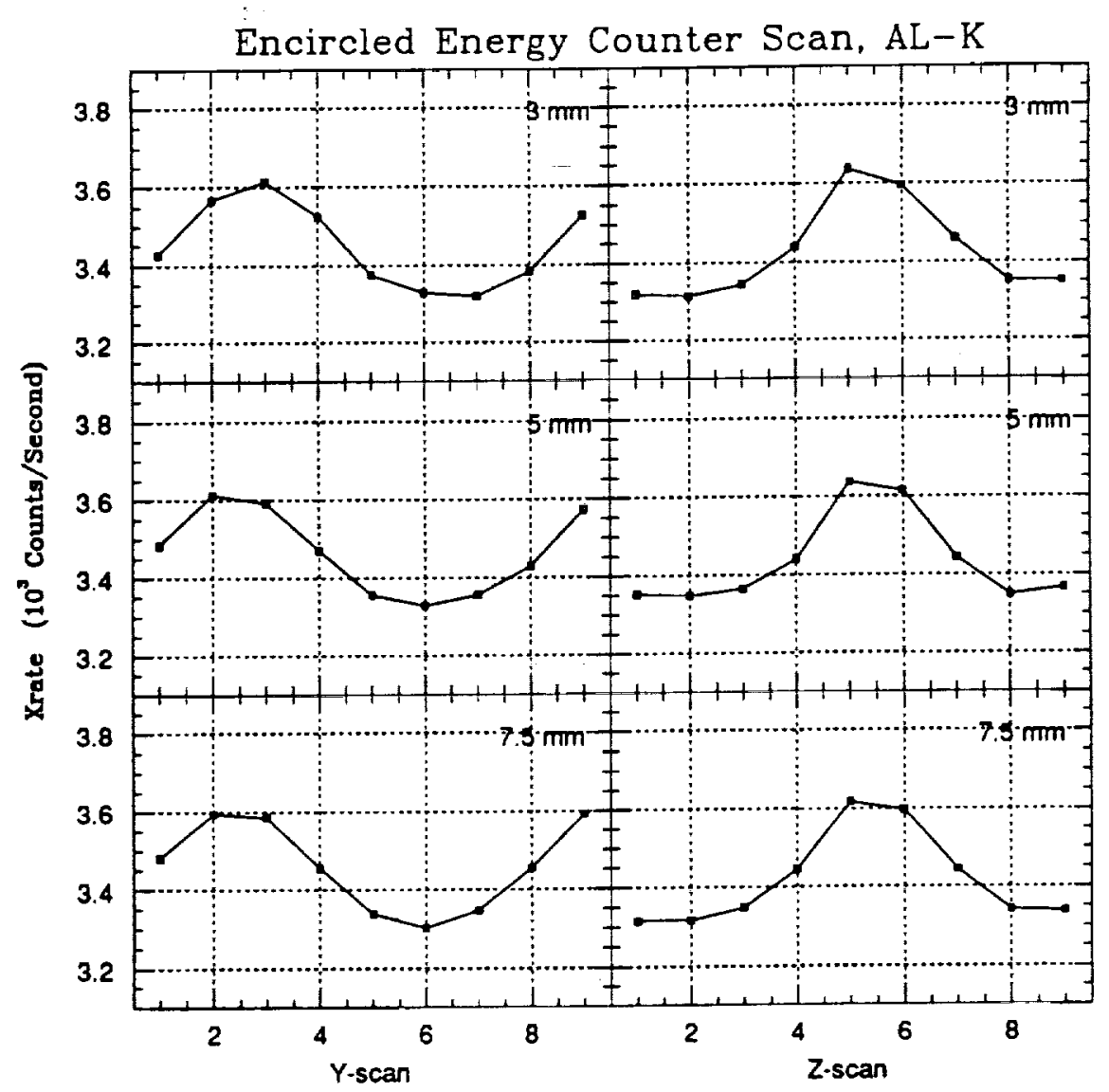

Figure 4: VETA Encircled Energy Window Scan Quick-look Data. Al-K source, aperture 3 - $7.5 \mathrm{~mm}$.

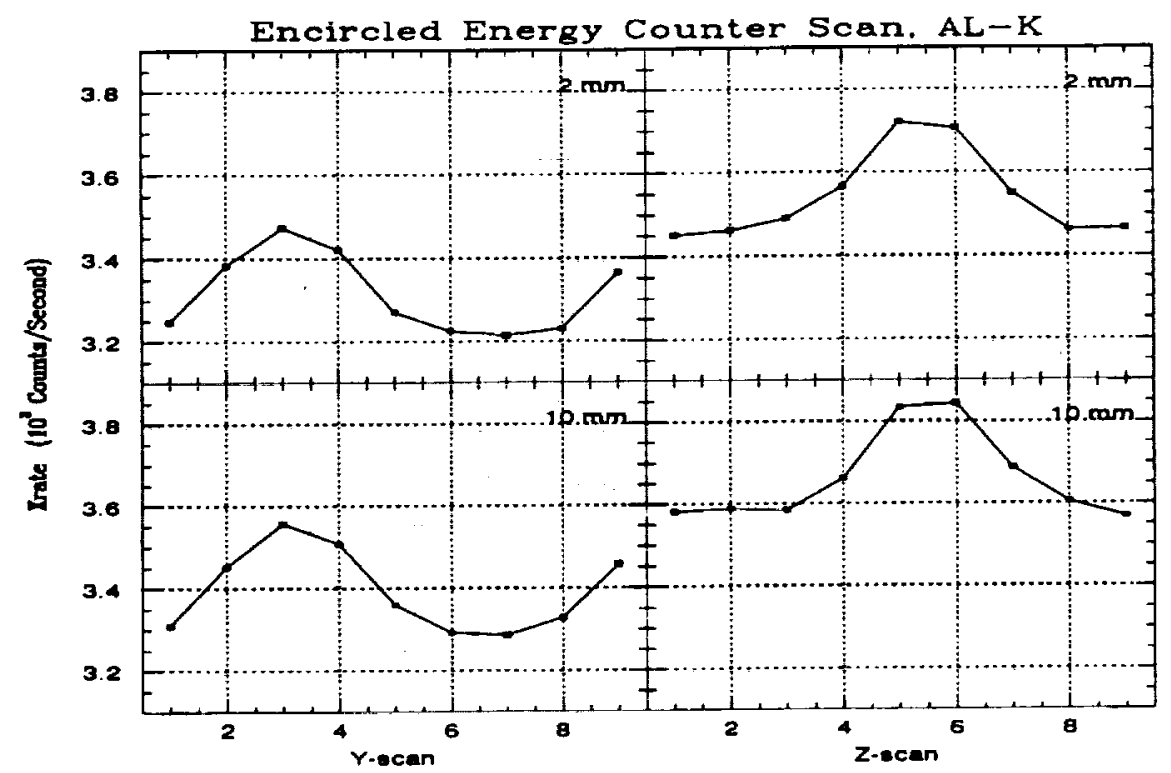

Figure 5: VETA Encircled Energy Window Scan Quick-look Data. Al-K source, aperture 2 and $10 \mathrm{~mm}$. The Z-scans have much higher counts than $\mathrm{Y}$-scans. It indicates that these window scan measurements were not done as planned. 


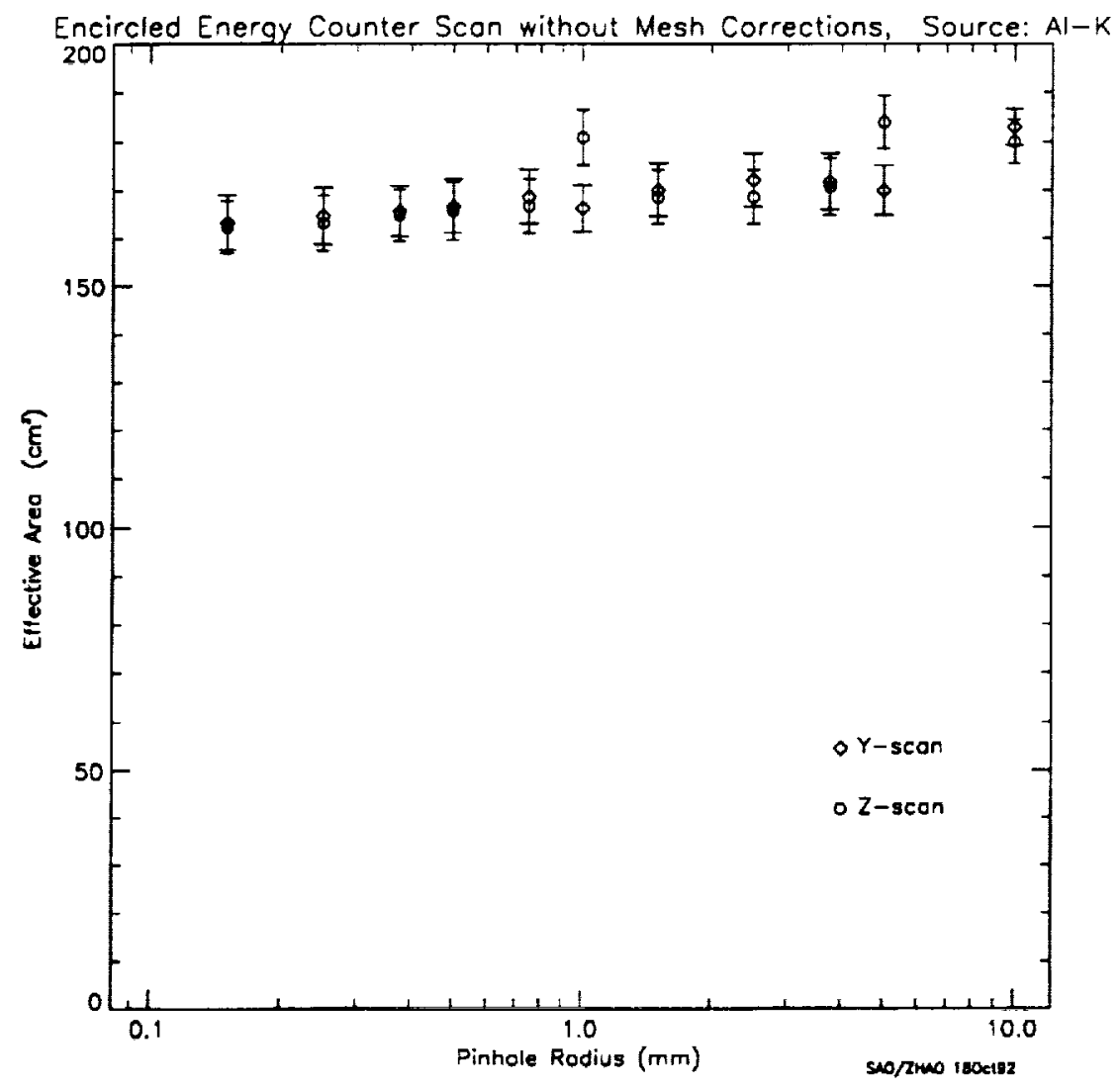

Figure 6: VETA Encircled Energy vs. aperture size Quick-look Data. Al-K source. The $20 \mathrm{~mm}$ aperture window scans and the Z-scans for the 2 and $10 \mathrm{~mm}$ aperture are distinctively higher than the rest of the window scans.

Measurement which was carried out in March of 1992 at XRCF.

\section{POST VETA MEASUREMENTS}

Two Post VETA Measurements were made. The first one was the mechanical measurement which solely supported the window mesh effect analysis. The second one was the X-ray measurement which measured the beam uniformity, filter thickness, detector response etc., in supporting the whole VETA data analysis and HRMA (High Resolution Mirror Assembly) test planning. In the mechanical measurement we measured: 1) the mesh grid periodicity; 2) the mesh wire orientation; 3) relative $Y$ and $Z$ positions between the VETA focal points and the wire mesh as well as the counter window bezel; 4) VETA focal point to wire mesh distance (along the $\mathrm{X}$ axis). Depending upon the region on the window, the mesh periodicity varies between $499 \mu \mathrm{m}$ to $564 \mu \mathrm{m}$. The mesh wires are laid within 0.2 degrees from the horizontal and vertical directions. A motor log recorded all the motor positions during the VETA test. We used this motor log to repeat all the moves and measured the aperture and counter positions. Figure 7 shows the actual positions on the window mesh where the $\mathrm{X}$-rays enter the window for different scans and apertures. It is seen that the $\mathrm{Y}$-scans and Z-scans were actually made at different parts of the window. There are actually six locations on the window where data were taken: window $Y$-scan, $Z$-scan and window fixed measurement for 2 and $10 \mathrm{~mm}$ apertures; window $Y$-scan, Z-scan and window fixed measurement for other apertures. There was a so called Prime-Y move, which moves the whole XDA assembly in order to reach different parts of the detector, during the VETA test. We later found this Prime-Y move had a $0.1 \%$ error - it was $100 \mu \mathrm{m}$ short for a $100 \mathrm{~mm}$ move - and also its repeatability was not very good. But our motor log regards every move as accurate as its read out. Therefore the positions shown in Figure 7 are only good to $\pm 20 \mu \mathrm{m}$. More accurate window positions 


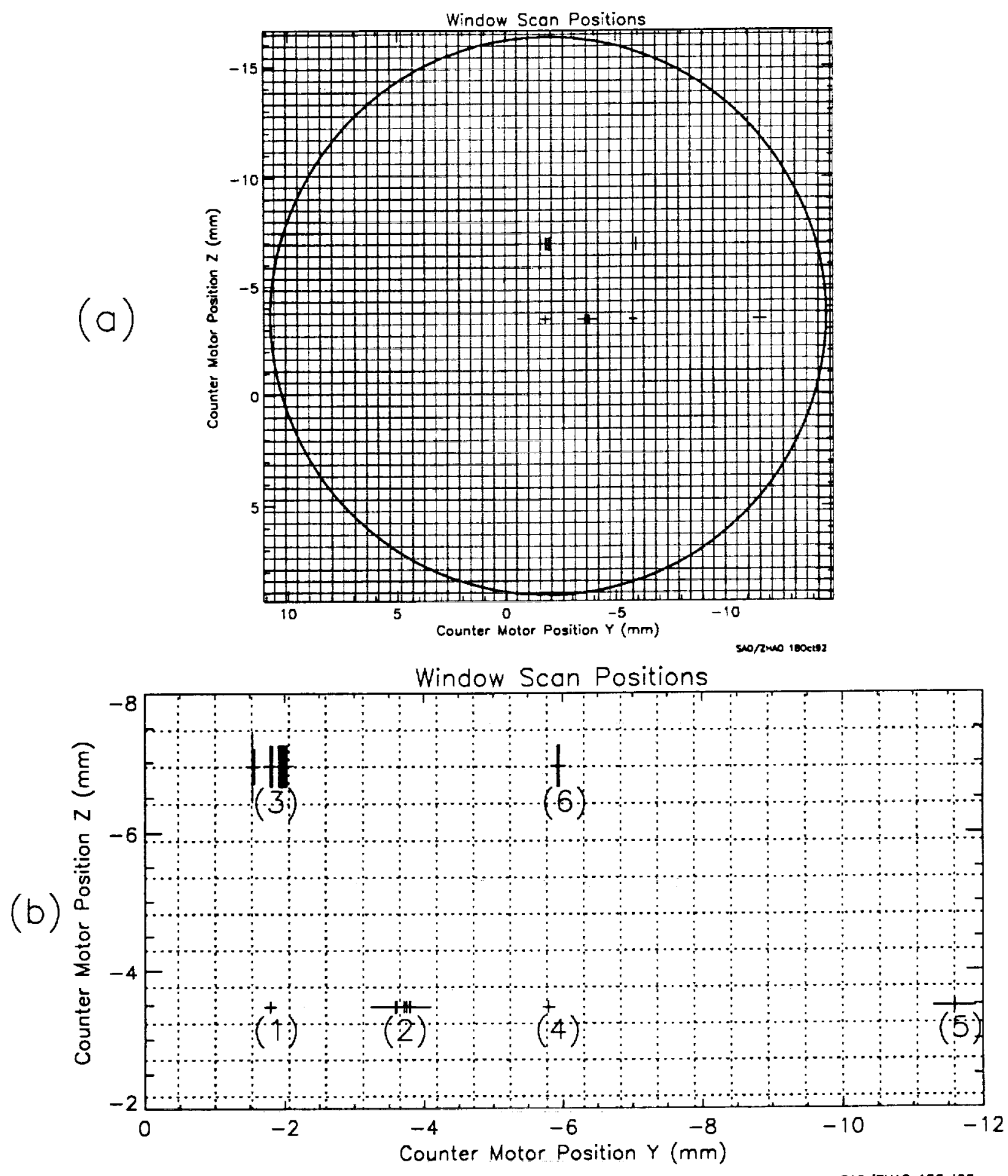

Figure 7: Window Scan Positions. A result of the Post VETA Measurements. (a) shows the relative positions of the window bezel (big circle), mesh grid and the aperture center positions where the measurements were made (little crosses). (b) shows a magnified portion of (a). The measurements were bunched at six locations. Location (4), (5) and (6) are for 2 and $10 \mathrm{~mm}$ apertures. Location (1), (2) and (3) are for the rest of the apertures. Window fixed measurements were made at locations (1) and (4). They were also for the planned window scan measurements. But the actual window $Y$-scan measurements were made at (2) and (5). The actual window Z-scan measurements were made at (3) and (6). 
were obtained by fitting the VETA data to the mesh models (see next two sections). Because the window slightly bulged out under the pressure, the VETA focal point (the center of the $5 \mu$ m aperture was used to refer to this point) to wire mesh distances were measured at all six positions shown in Figure 7 under 125 and 400 torr differential pressures. The results are in Table 1.

Table 1. VETA Focus to Window Mesh Distance

\begin{tabular}{||c|c|c|c|c||}
\hline Source & Gas \& & \multicolumn{3}{|c||}{ Aperture Size } \\
\cline { 3 - 6 } & Pressure & $0.005-7.5 \mathrm{~mm}$ & $2 \& 10 \mathrm{~mm}$ & $20 \mathrm{~mm}$ \\
\hline $\mathrm{C}$ & Methane/125 Torr & $24.940 \mathrm{~mm}$ & $24.940 \mathrm{~mm}$ & $29.012 \mathrm{~mm}$ \\
\hline $\mathrm{Al}, \mathrm{Cu}, \mathrm{Mo}, \mathrm{Zr}$ & P10/400 Torr & $24.703 \mathrm{~mm}$ & $24.814 \mathrm{~mm}$ & $28.775 \mathrm{~mm}$ \\
\hline
\end{tabular}

\section{WIRE MESH COMPUTER MODELS}

Computer models of the window mesh were developed to simulate the X-ray transmission based on the exact XDA geometry and our knowledge of the VETA. ${ }^{7}$ First, ray-trace image files of X-ray on the counter window were generated by using the OSAC package. The ray-trace included the effects of residual gravitational distortions, scatterings for different energies, despace due to the uncut glass, apodization, finite source sizes and their intensity distribution, finite source distance, mirror surface figures, and mirror support strut. For all five sources and different focuses to mesh distances, there are 14 ray-trace image files generated and each contains 50000 photons. We then laid each ray-trace image on top of a mesh wire grid model and calculated the number of photons blocked by the wire grid. The mesh grid model was then moved in both $\mathrm{Y}$ and $\mathrm{Z}$ directions with the ray-trace image fixed to simulate the window scan, and transmission was calculated for each move. Figure 8 shows the ray-trace image of the Al-K source on top of the wire grid for one fixed position. Figure 9 shows the mesh transmission model for the Al-K source with a 2-dimensional scan of $81 \times 81$ positions. It is seen that the mesh effect is very strong. For $20 \mathrm{~mm}$ aperture, the transmission is the minimum when the center of the aperture is at one of the intersections of the mesh grid. For other apertures, the transmission is the maximum in that case. Depending on the counter position, the X-ray transmission can vary between $75 \%$ and $92 \%$. There are 14 such mesh models corresponding to 14 ray-trace files as mentioned above.

\section{WIRE MESH CORRECTIONS}

To make the mesh effect correction, we had to first fit the data to the mesh model to find the accurate $\mathrm{Y}$ and $\mathrm{Z}$ positions of the window scan. The correction was then made by comparing the percentage of transmission at these positions in the mesh model to the BND counter mesh transmission. The quick-look data shown in section 3 provide a preliminary result during and immediately after the VETA test. The spectrum fitting analysis was done for all the VETA encircled energy data to correct spectral contamination including bremsstrahlung continuum, pulse pileup, background and deadtime. ${ }^{6}$ The spectrum corrected data were then used to fit with their corresponding mesh models with a minimum $\chi^{2}$. The initial fit was made based on the window scan positions measured during the post VETA measurement. The VETA motor log gives the relative positions between $\mathrm{Y}$-scan and $\mathrm{Z}$-scan for each aperture. More accurate fit was then obtained by combining the initial fit and the motor log. This process was like fitting a two dimensional data to the mesh model, even though the data were only from two one dimensional scans perpendicular to each other. The data fit the mesh model very well. The reduced $\chi^{2}$ for each window $Y$ and $Z$ scan pair fit ranges from 0.4 to 1.4 with the average around 1. Figure 10 (a) and (b) show one of the typical fits. The fitting process located the exact relative positions between the aperture and the window mesh. The XDA transmission rate at these positions was then calculated using the mesh model. The mesh corrections were finally made by multiplying the spectrum corrected data with the BND transmission rate $(81.72 \%)$ and dividing it by the calculated XDA transmission rate. Figure 10 (c) and (d) show the corrected encircled energy window scan data. It is seen that the mesh modulation effect is removed. 



Figure 8: Raytrace Image on Mesh grid Model. Al-K source. (a) and (b) show, one fixed counter position, the 50000 photon raytrace images on top of the mesh grin model for $0.005-10 \mathrm{~mm}$ apertures and $20 \mathrm{~mm}$ aperture, respectively. (c) and (d) show the mesh transmission pattern for the same raytrace images. 

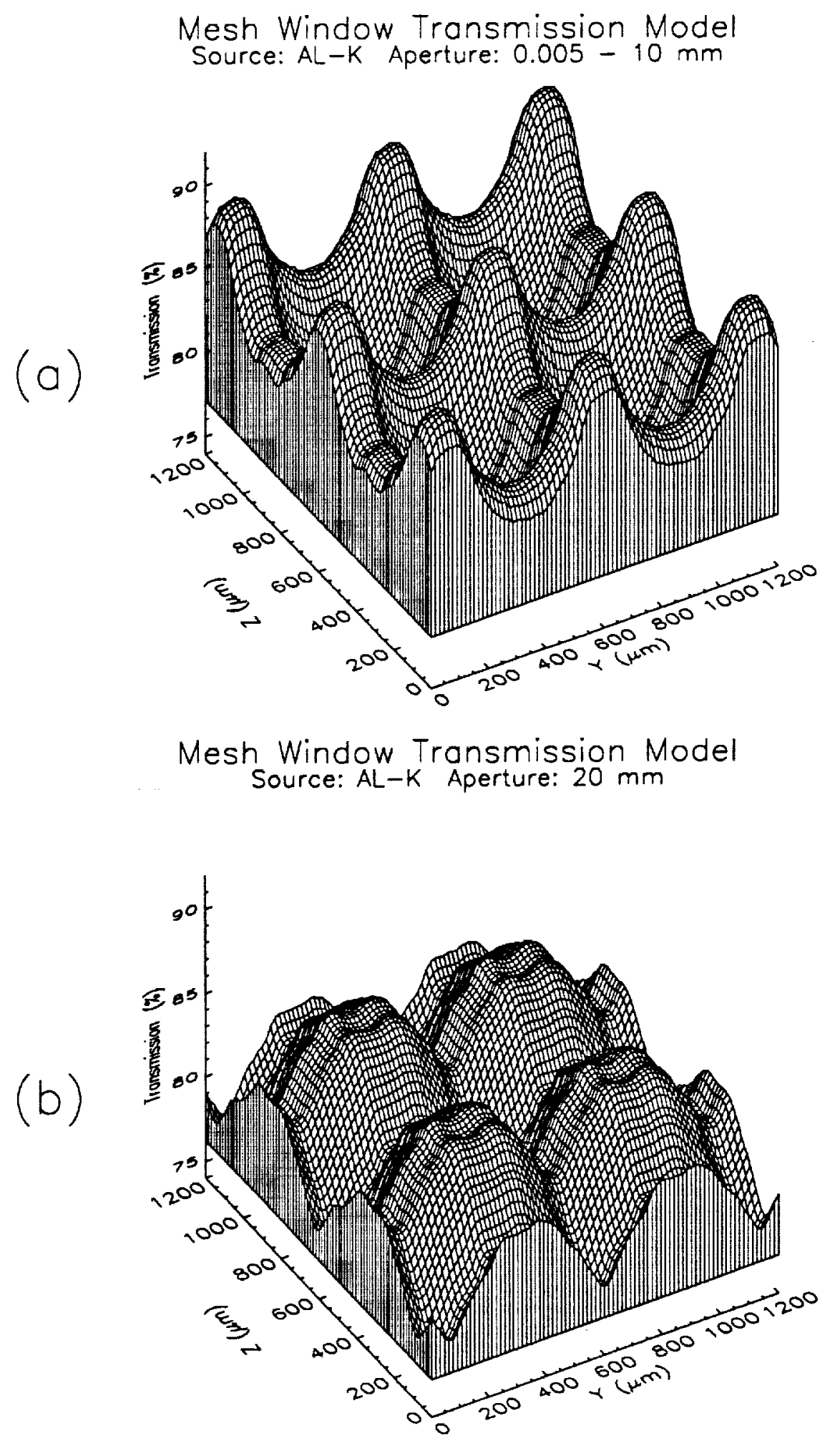

Figure 9: Window Mesh Transmission Models. Al-K source. (a) is the model for 0.005 to $7.5 \mathrm{~mm}$ apertures, in which a maximum transmission occurs when the $Y-Z$ coordinates of the aperture center is at a mesh wire intersection. (b) is the model for $20 \mathrm{~mm}$ aperture, in which a minimum transmission occurs when the Y-Z coordinates of the aperture center is at a mesh wire intersection. Depending on the counter position, the $\mathrm{X}$-ray transmission can vary between $75 \%$ and $92 \%$. 
Window Scan Dolo fitted with the Mesh Tronsmission Model Madel file: mesh537_E 2.07_FDIST_24703

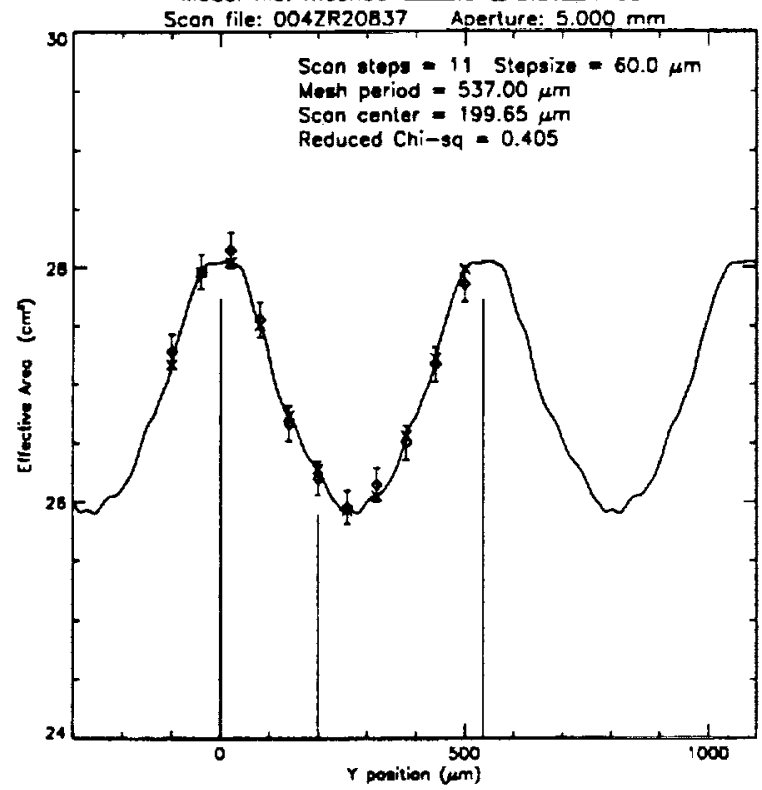

Window Scon Dota with Mesh Transmission Corrections Window Scon file: 004ZR20B37

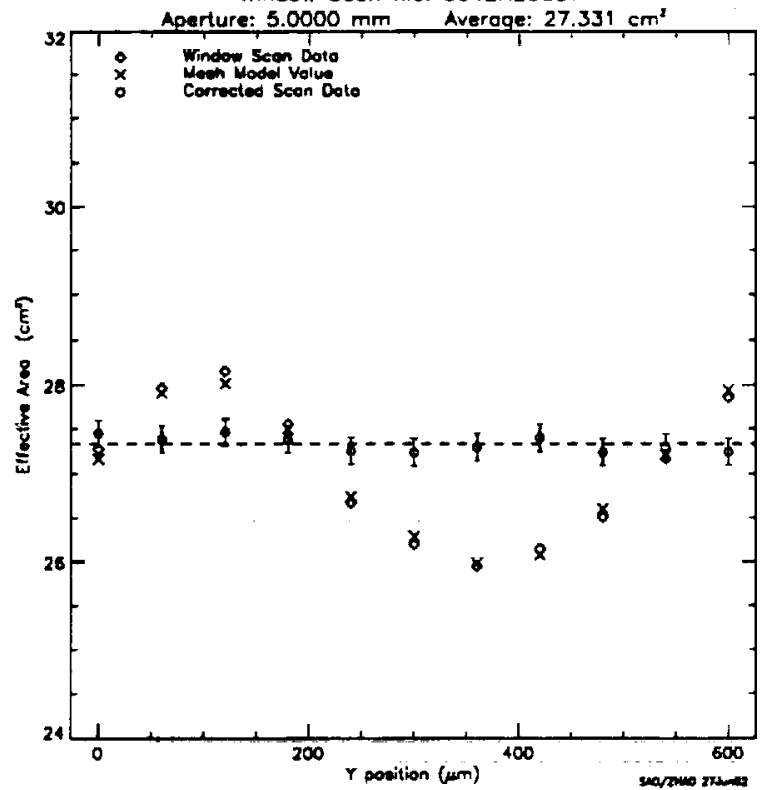

Window Scon Doto fitted with the Mesh Transmission Model Window Scon Doto fitted with the Mesh Transmissi

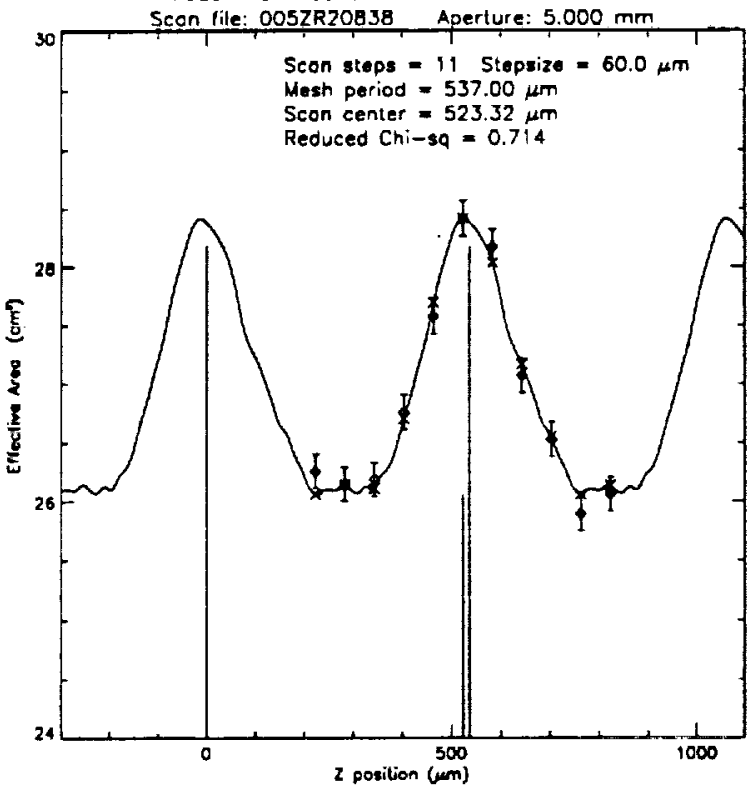

Window Scan Dato with Mesh Transmission Corrections Window Scan file: 005ZR20838

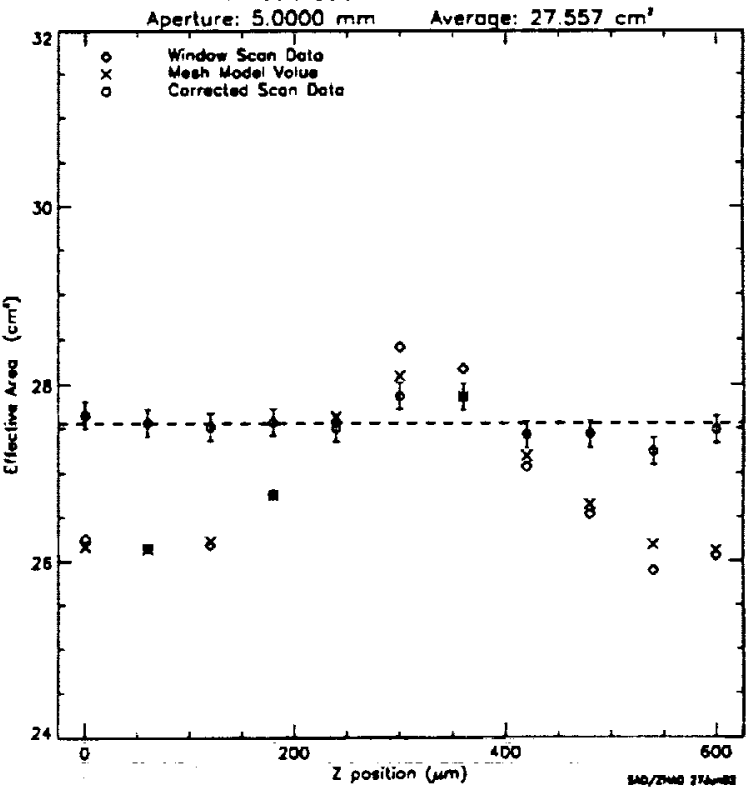

Figure 10: A Typical Window Scan Data Fit to The Mesh Model. Zr-L source, $5 \mathrm{~mm}$ aperture. Top two figures show the $\mathrm{Y}$ and $\mathrm{Z}$ scan data fit to the mesh model. Bottom two figures show the same data after the mesh correction. The mesh modulation effect is removed. 
The window scan measurement only covered three sources ( $\mathrm{Al}, \mathrm{C}$ and $\mathrm{Zr}$ ) and 11 apertures $(0.3 \mathrm{~mm}$ through $20 \mathrm{~mm}$ ). Before the window scan measurements, we did the window fixed measurement for all five sources and 16 apertures $(0.005$ through $20 \mathrm{~mm})$. Instead of scanning the counter, only one fixed counter position was used for each aperture. In order to make mesh effect corrections for the window fixed measurements, we had to know their exact counter positions. We do have a motor log which gives the relative counter positions between all the window scan and window fixed measurements. However, there were prime-Y moves during the measurement which make the motor log not very reliable. But we did the beam centering for apertures ranging from $0.005 \mathrm{~mm}$ to $0.3 \mathrm{~mm}$. The $0.3 \mathrm{~mm}$ aperture was the only one used for both window scan and beam centering thus it linked the window fixed data and the window scan data. There was no or very small Prime-Y move between 0.005 and $0.3 \mathrm{~mm}$ aperture window fixed measurements. With this information, we were able to accurately locate the counter positions for apertures from 0.005 $\mathrm{mm}$ to $0.3 \mathrm{~mm}$ window fixed measurements and hence to make their mesh effect corrections. For the final results, we use the window scan data for 0.3 to $20 \mathrm{~mm}$ apertures and window fixed data for 0.005 to $0.1 \mathrm{~mm}$ apertures. Because there was no window scan measurement for $\mathrm{Cu}$ and Mo sources, we could only rely on our motor log to make some rough mesh corrections for these two sources. Therefore the results for these two sources have bigger errors.

\section{FINAL RESULTS OF THE VETA-I ENCIRCLED ENERGY}

Table 2 gives the final results and their errors for the VETA encircled energy. The final errors are less

Table 2. VETA-I Encircled Energy units: $\mathrm{cm}^{2}$

\begin{tabular}{||c|c|c|c|c|c||}
\hline \multirow{2}{*}{$\begin{array}{c}\text { Aperture } \\
\text { size (mm) }\end{array}$} & \multicolumn{5}{|c||}{ X-ray lines } \\
\cline { 2 - 6 } & $\begin{array}{c}\mathrm{C}-\mathrm{K} \\
0.277 \mathrm{keV}\end{array}$ & $\begin{array}{c}\mathrm{Cu}-\mathrm{L} \\
0.932 \mathrm{keV}\end{array}$ & $\begin{array}{c}\mathrm{Al}-\mathrm{K} \\
1.488 \mathrm{keV}\end{array}$ & $\begin{array}{c}\mathrm{Zr}-\mathrm{L} \\
2.067 \mathrm{keV}\end{array}$ & $\begin{array}{c}\text { Mo-L } \\
2.334 \mathrm{keV}\end{array}$ \\
\hline 0.005 & 4.375 & 3.362 & 2.959 & 0.516 & 0.155 \\
\hline 0.010 & 8.839 & 8.471 & 7.498 & 1.352 & 0.349 \\
\hline 0.025 & 31.651 & 24.662 & 24.302 & 3.636 & 0.954 \\
\hline 0.050 & 65.126 & 49.312 & 49.001 & 7.270 & 2.129 \\
\hline 0.100 & 123.797 & 97.395 & 94.425 & 14.521 & 4.271 \\
\hline 0.300 & 214.293 & 167.884 & 165.682 & 24.939 & 7.784 \\
\hline 0.750 & 217.538 & 181.863 & 166.690 & 25.664 & 8.011 \\
\hline 0.500 & 220.655 & 186.698 & 168.720 & 26.085 & 7.987 \\
\hline 1.000 & 217.422 & 179.857 & 169.617 & 26.207 & 8.164 \\
\hline 1.500 & 219.620 & 180.507 & 170.602 & 26.536 & 8.233 \\
\hline 2.000 & 217.813 & 178.935 & 170.995 & 27.119 & 8.374 \\
\hline 3.000 & 222.311 & 186.224 & 173.035 & 26.982 & 8.999 \\
\hline 5.000 & 220.197 & 174.568 & 173.674 & 27.445 & 9.040 \\
\hline 7.500 & 221.697 & 190.402 & 174.769 & 27.691 & 9.272 \\
\hline 10.000 & 219.958 & 185.656 & 174.259 & 27.804 & 8.772 \\
\hline 20.000 & 223.037 & 182.731 & 179.007 & 28.706 & 8.497 \\
\hline \hline Total Error & $0.562 \%$ & & $0.612 \%$ & $0.795 \%$ & \\
\hline Error due to & $0.422 \%$ & & $0.470 \%$ & $0.772 \%$ & \\
\hline Mesh Correction & & & & & \\
\hline
\end{tabular}

than $\pm 0.8 \%$ for $\mathrm{Al}, \mathrm{C}$ and $\mathrm{Zr}$ sources, which exceeded the requirements $( \pm 2 \%)$ by a factor of 2.5 . Figures 11 , 12 and 13 are the plots of the encircled energy vs. the aperture sizes before and after the mesh corrections for $\mathrm{Al}, \mathrm{C}$ and $\mathrm{Zr}$ sources. These results were then used to compare with the expected values to get the VETA point spread functions and the mirror surface figures. ${ }^{7}$ they were also used to obtain the VETA total effective area and mirror reflectivity. ${ }^{5}$ 

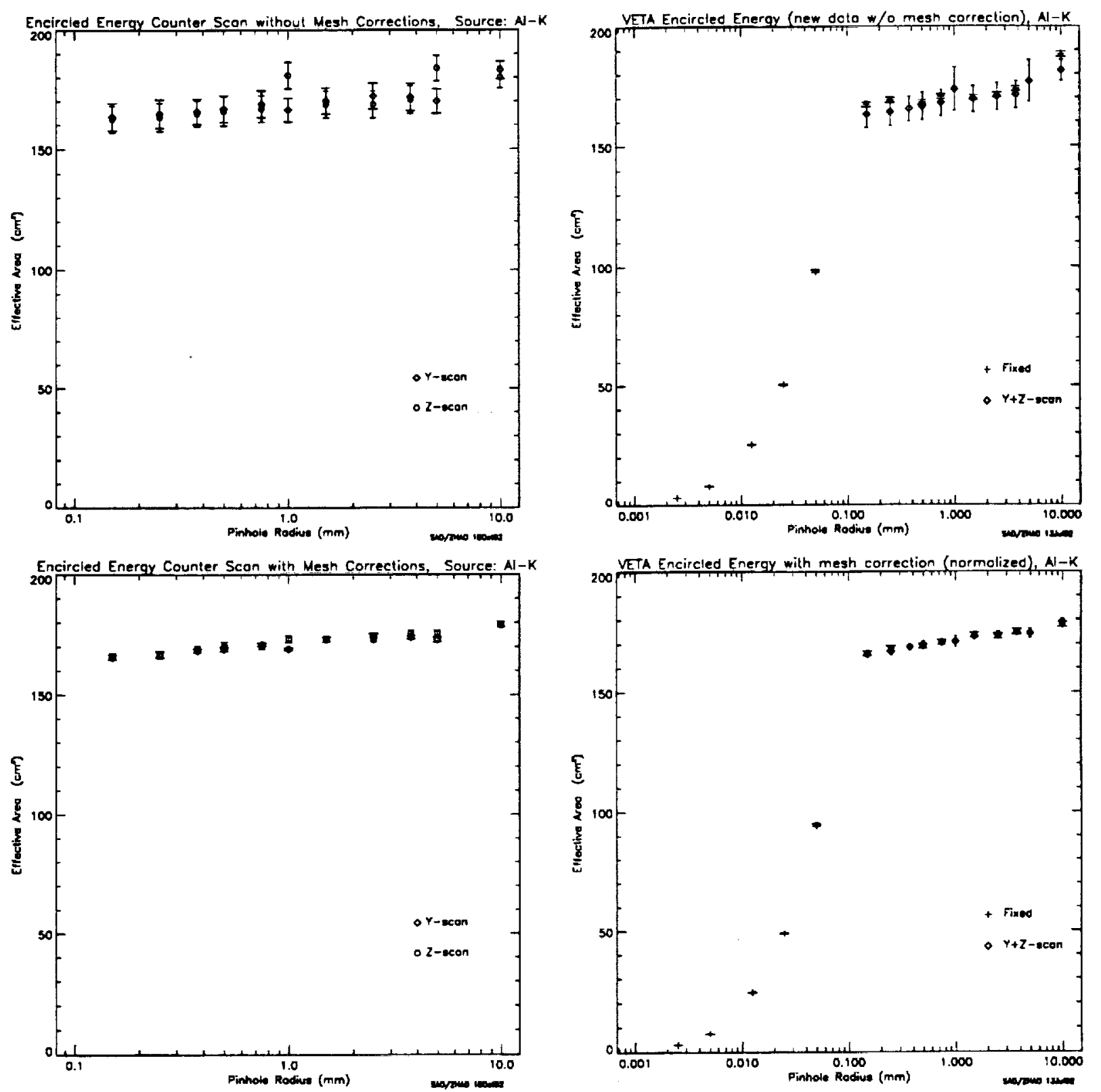

Figure 11: The VETA-I Encircled Energy before (above) and after (below) the Mesh Corrections. Al-K source. 

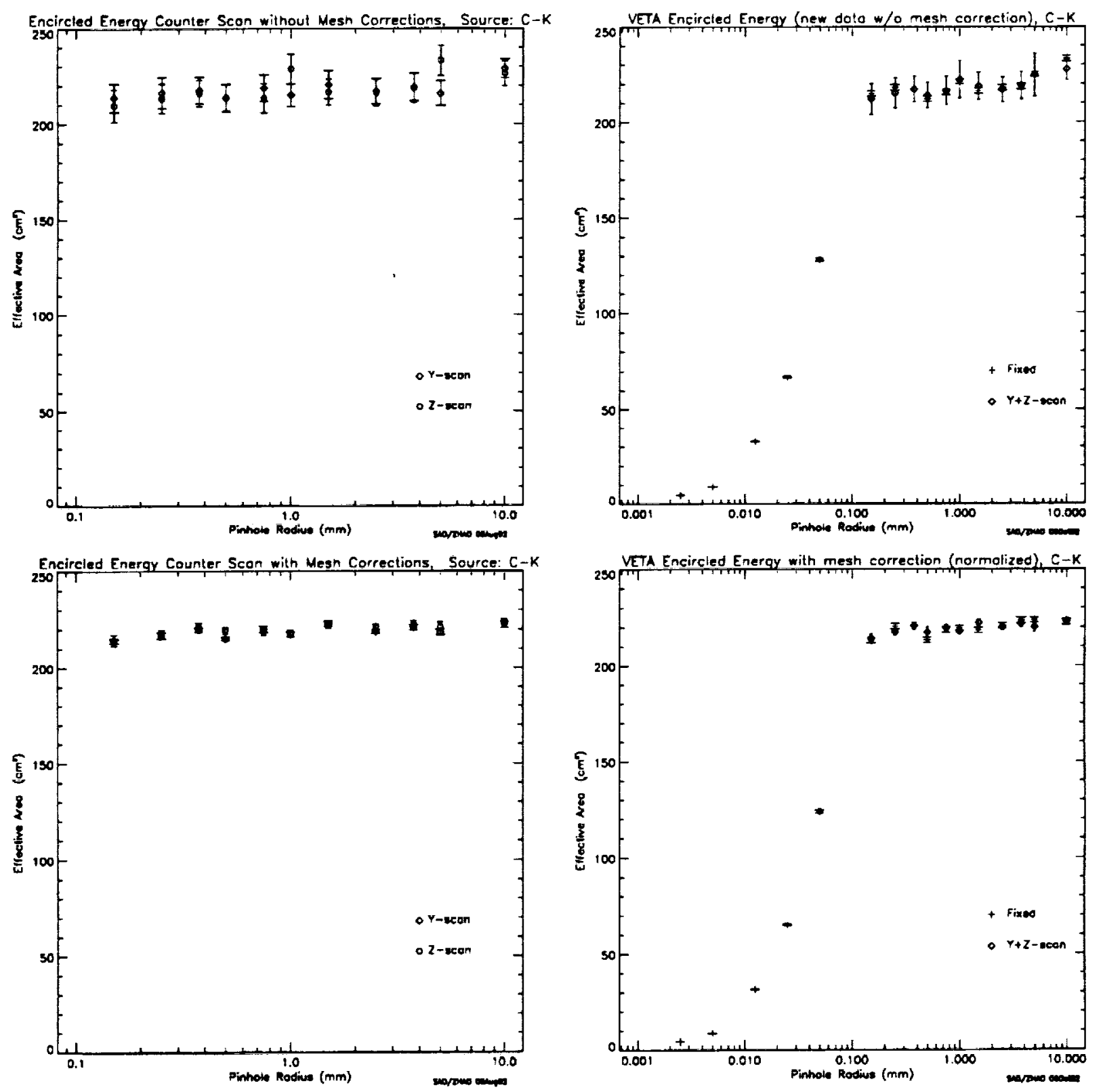

Figure 12: The VETA-I Encircled Energy before (above) and after (below) the Mesh Corrections. C-K source. 

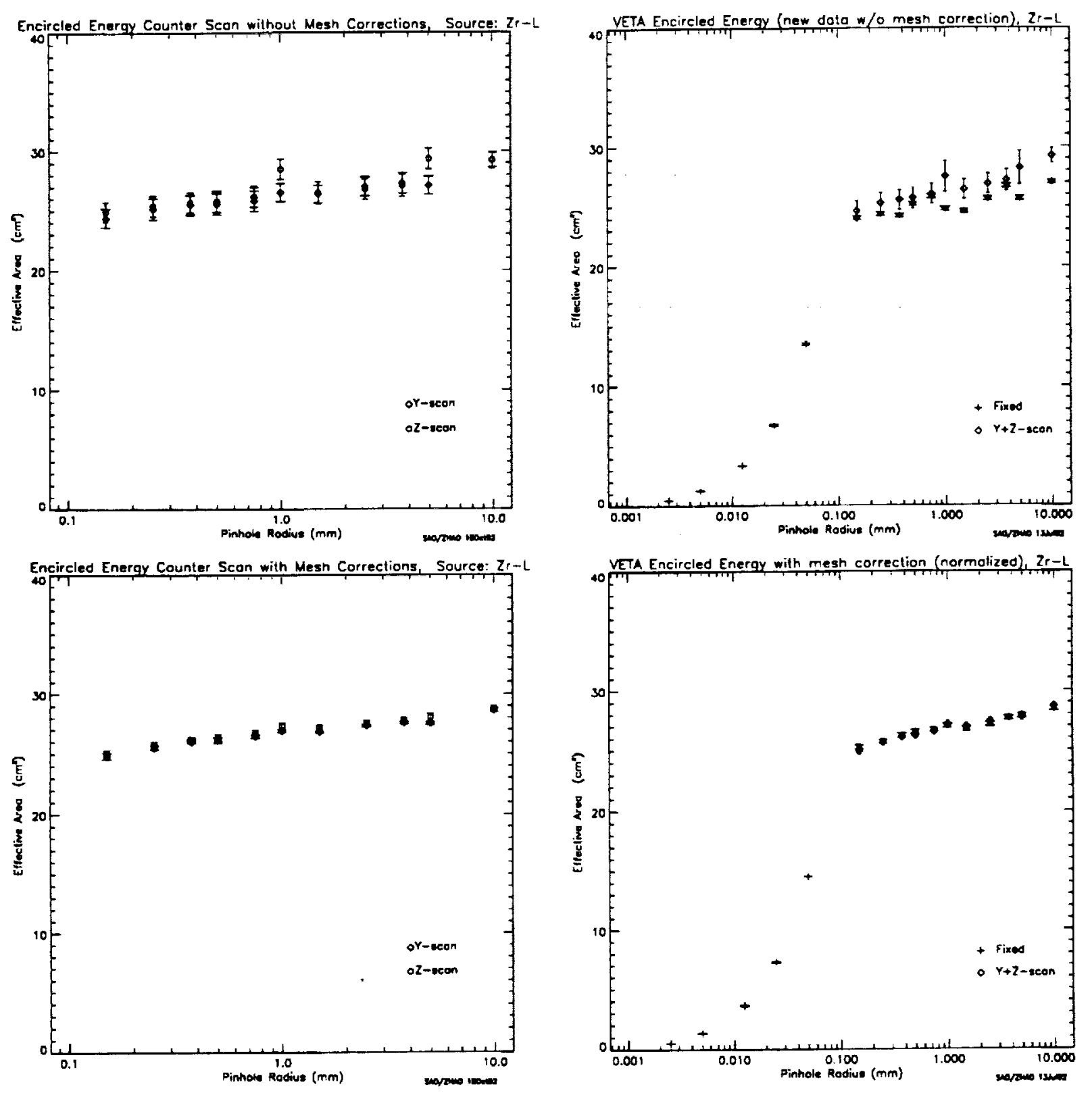

Figure 13: The VETA-I Encircled Energy before (above) and after (below) the Mesh Corrections. $\mathrm{Zr}-\mathrm{L}$ source. 


\section{ACKNOWLEDGMENT}

We would like to thank Roger Brissenden, George Chartas, Mark Jones, Tim Norton, William Podgorski, John Roll, and Dan Schwartz for their help during the course of this work. This work was supported under NASA contract \# NAS8-36123.

\section{REFERENCES}

1. M. C. Weisskopf, "The Advanced Astrophysics Facility: An Overview," Astrophysical Letters $E$ Communications, Vol. 26, pp. 1-6, 1987.

2. E. M. Kellogg, R. J. V. Brissenden, K. A. Flanagan, M. D. Freeman, J. P. Hughes, M. T. Jones, M. Ljungberg, P. Mckinnon, W. A. Podgorski, D. A. Schwartz, and M. V. Zombeck, "Calibration of the Verification Engineering Test Article-I (VETA-I) for AXAF using the VETA-I X-ray Detection System," Proc. SPIE, Vol. 1546, pp. 2-12, 1991.

3. P. Zhao, E. M. Kellogg, D. A. Schwartz, Yibo Shao, and M. A. Fulton, "Intensity distribution of the $\mathrm{X}$-ray source for the AXAF VETA-I mirror test," Proc. SPIE, this volume, 1992.

4. W. A. Podgorski, K. A. Flanagan, M. D. Freeman, R. G. Goddard, E. M. Kellogg, T. J. Norton, J. P. Ouellette, A. G. Roy, and D. A. Schwartz, "VETA-I X-ray detection system," Proc. SPIE, this volume, 1992.

5. E. M. Kellogg, G. Chartas, D. Graessle, J. P. Hughes, L. Van Speybroeck, P. Zhao, M. C. Weisskopf, R. F. Elsner, and S. L. O'Dell, "The X-ray reflectivity of the AXAF VETA-I optics," Proc. SPIE, this volume, 1992.

6. G. Chartas, K. A. Flanagan, J. P. Hughes, E. M. Kellogg, D. Nguyen, M. V. Zombeck, M. Joy, and J. J. Kolodziejczak, "Correcting X-ray spectra obtained from the AXAF VETA-I mirror calibration for pileup, continuum, background and deadtime," Proc. SPIE, this volume, 1992.

7. J. P. Hughes, D. A. Schwartz, A. Szentgyorgyi, L. Van Speybroeck, and P. Zhao, "Surface finish Quality of the Outer AXAF mirror pair based on X-ray measurement of the VETA-I," Proc. SPIE, this volume, 1992. 